\title{
Direct simulation of extinction in a slab of spherical particles
}

\author{
D.W. Mackowski ${ }^{\text {a,*}}$, M.I. Mishchenko ${ }^{\text {b }}$ \\ a Department of Mechanical Engineering, Auburn University, Auburn, AL 36849, USA \\ b NASA Goddard Institute for Space Studies, 2880 Broadway, New York, NY 10025, USA
}

\section{A R T I C L E I N F O}

\section{Article history:}

Received 5 November 2012

Received in revised form

6 February 2013

Accepted 11 February 2013

Available online 1 March 2013

Keywords:

Electromagnetic scattering

Random media

Radiative transfer

\begin{abstract}
A B S T R A C T
The exact multiple sphere superposition method is used to calculate the coherent and incoherent contributions to the ensemble-averaged electric field amplitude and Poynting vector in systems of randomly positioned nonabsorbing spherical particles. The target systems consist of cylindrical volumes, with radius several times larger than length, containing spheres with positional configurations generated by a Monte Carlo sampling method. Spatially dependent values for coherent electric field amplitude, coherent energy flux, and diffuse energy flux, are calculated by averaging of exact local field and flux values over multiple configurations and over spatially independent directions for fixed target geometry, sphere properties, and sphere volume fraction. Our results reveal exponential attenuation of the coherent field and the coherent energy flux inside the particulate layer and thereby further corroborate the general methodology of the microphysical radiative transfer theory. An effective medium model based on plane wave transmission and reflection by a plane layer is used to model the dependence of the coherent electric field on particle packing density. The effective attenuation coefficient of the random medium, computed from the direct simulations, is found to agree closely with effective medium theories and with measurements. In addition, the simulation results reveal the presence of a counter-propagating component to the coherent field, which arises due to the internal reflection of the main coherent field component by the target boundary. The characteristics of the diffuse flux are compared to, and found to be consistent with, a model based on the diffusion approximation of the radiative transfer theory.

(c) 2013 Elsevier Ltd. All rights reserved.
\end{abstract}

\section{Introduction}

Modern, parallel-based computational hardware, coupled with superposition methods for calculating the net scattered field by a collection of particles, have made feasible the use of direct simulation strategies to investigate the characteristics of electromagnetic energy transport in dense particulate systems. A direct simulation, by definition, provides an exact description of the

\footnotetext{
* Corresponding author. Tel.: +1 334844 3334;

fax: +1 3348443307 .

E-mail address: mackodw@auburn.edu (D.W. Mackowski).
}

electromagnetic field both within and external to a target containing a large yet finite number of particles, and which is excited by an external source of radiation. In this sense, results generated by direct simulations can be considered a benchmark for evaluation of analytical or phenomenological theories to describe the propagation of electromagnetic energy in discretely inhomogeneous media. As a case in point, we have recently used the multiple sphere $T$ matrix (MSTM) code to calculate the scattering matrices of targets containing up to several thousand spheres, with the individual spheres having size parameters up to 4 [1-3]. The targets, in these calculations, consisted of spherical volumes, with the spheres randomly distributed within the volume with a set overall 
volume fraction; volume fractions used in the calculations ranged from 0.01 to 0.4 . Since the number of spheres in the targets was large, we assumed that the configurationaveraged scattering matrix (i.e., that obtained from averaging scattering matrices, each obtained for a fixed realization of the sphere properties and a fixed incident direction) could be approximated by the orientation averaged scattering matrix for a single realization; this allowed us to exploit the analytical orientation averaging properties of the target $T$ matrix as calculated by MSTM. Results from these calculations have provided definitive evidence to support microphysical theories of radiative transfer and coherent backscattering [4].

The objective of this work is to present a similar comparison between numerically exact computer simulations and analytical theory. The phenomena under examination, in this case, are the attenuation of the coherent field, and the propagation of the coherent and diffuse components to the time-averaged Poynting vector in a particulate medium, due to scattering by the particles. The simulation procedure is relatively straightforward: local electric and magnetic field amplitudes will be calculated for a statistically representative sequence of randomly generated targets of spheres, with each target configuration corresponding to the same sphere properties and average concentration. The local coherent field and time-averaged Poynting vector are then obtained by assuming ergodicity and averaging over the sequence of configurations. An important difference in the procedures used here, as opposed to our previous investigations [2,3], is that they will not employ analytical $T$ matrix averaging: our focus is on the local field amplitudes within the medium as opposed to the far-field scattering behavior.

A well established theoretical framework has been developed to describe coherent field attenuation in discretely inhomogeneous media. On the most basic level is the standard radiative transfer theory, which represents the dilute concentration limit $[2,4]$. The imaginary part of the effective propagation constant in the medium, for this approximation, is obtained from the product of the particle number density and average extinction cross section.

The radiative transfer theory is based on the far-field version of the Foldy-Lax equations [4] and will fail for wavelength-sized particles with concentrations approaching and exceeding 0.1 . For such cases comprehensive effective medium (EM) formulations have been developed, most notably by Ishimaru and the Varadans [5-7]. Such theories begin with the same basic superposition model employed in the direct simulation, for which the exciting field at a particular sphere is given by the incident (i.e., externally exciting) field and the sum of fields scattered from every other sphere in the system. Unlike a direct simulation - which solves the superposition equations for each sphere in the system and then averages over multiple configurations - EM formulations attempt to average the superposition equations analytically, with subsequent derivation of relations for the effective propagation constant. Performing this averaging requires knowledge of the pair correlation function of the particles in the random medium, and it also invokes simplifying assumptions regarding the high-order correlations among the particle positions and scattered fields, e.g., the quasi-crystalline approximation (QCA) [8-10].

Both computational and experimental evidence has been collected to validate EM theories. Tsang et al. [11] inferred the attenuation rate from direct simulations of far-field scattering by a particulate volume and obtained good correspondence with theory, although the simulations were limited to spheres with a relatively small size parameter of 0.2. Analytical estimates of EM theories have been shown to accurately predict laboratory measurements of extinction in random suspensions of monodisperse spheres having size parameters of order unity or greater [12].

The investigations in $[11,12]$ were focussed entirely on the effective propagation constant (or, equivalently, effective refractive index) of the spherical-particle system. As predicted by theory, this quantity depends solely on the sphere properties, concentration, and pair correlation function; it is not a function of the geometry of the system containing the particles. Unlike Ref. [11], the objective of this work is to examine how the target geometry affects the coherent field within the target. In particular, we will employ targets which model a fixed-thickness slab of particles. In an EM model, such a system would represent a plane layer. It is well known that interference of the forward and backward-propagating plane waves in a homogeneous plane layer can strongly affect the reflectance and transmission of the layer. We will show that the coherent field, in a slab of random spherical particles, can also cause interference effects.

An additional objective of this work is to examine the character of electromagnetic energy transport in the random medium. In particular, the direct simulations, combined with configurational averaging, will be used to calculate the coherent and diffusive components to the time-averaged Poynting vector in the medium. We will apply the diffusion approximation of the radiative transfer theory to model the diffuse flux.

\section{Methodology}

\subsection{Basic definitions}

We assume that the time dependence of the electric and magnetic fields is harmonic and described, in the complex-field representation, by the simple complex exponential $\exp (-\mathrm{i} \omega t)$, where $\omega$ is the angular frequency, $t$ is time, and $\mathrm{i}=\sqrt{-1}$. In other words, we assume that the complex electric and magnetic fields can be factorized as $\mathbf{E}(\mathbf{r}, t)=\exp (-\mathrm{i} \omega t) \mathbf{E}(\mathbf{r})$ and $\mathbf{H}(\mathbf{r}, t)=\exp (-\mathrm{i} \omega t) \mathbf{H}(\mathbf{r})$ respectively, where $\mathbf{r}$ is the position (radius) vector, while the actual real-valued fields are obtained by talking the real part of the respective complex fields. The complex field amplitudes $\mathbf{E}(\mathbf{r})$ and $\mathbf{H}(\mathbf{r})$ are constant if the scattering target is fixed, but vary with time implicitly if the scattering object undergoes temporal changes. In what follows, we will assume that temporal fluctuations of $\mathbf{E}(\mathbf{r})$ and $\mathbf{H}(\mathbf{r})$ caused by changes in the scattering object occur much more slowly than the harmonic oscillations of the factor $\exp (-\mathrm{i} \omega t)$. 
It is well known (see, e.g., Ref. [4]) that the instantaneous direction and rate of the electromagnetic energy flow at $\mathbf{r}$ are described by the Poynting vector $\mathbf{S}(\mathbf{r}, t)=\operatorname{Re}[\mathbf{E}(\mathbf{r}, t)] \times \operatorname{Re}[\mathbf{H}(\mathbf{r}, t)]$. Averaging the Poynting vector over a time interval capturing a sufficiently large number of time-harmonic oscillations of the electromagnetic field yields $\langle\mathbf{S}(\mathbf{r}, t)\rangle=\operatorname{Re}[\mathbf{S}(\mathbf{r})]$, where the complex Poynting vector is defined by $\mathbf{S}(\mathbf{r})=(1 / 2) \mathbf{E}(\mathbf{r}) \times \mathbf{H}^{*}(\mathbf{r})$ and the asterisk denotes a complex-conjugate value.

Let us consider the scattering object in the form of a volume of discrete random medium. The statistical randomness of this object is caused by chaotic movements of the participating particles resulting in relatively slow temporal fluctuations of the complex field amplitudes $\mathbf{E}(\mathbf{r})$ and $\mathbf{H}(\mathbf{r})$. At each given moment these amplitudes can be computed by solving the frequency-domain Maxwell equations for the corresponding instantaneous multiparticle configuration. In the majority of practical applications, one needs to calculate the average of a specific optical observable such as $\mathbf{S}(\mathbf{r}, t)$ over a time interval typically required to take an actual measurement. In what follows, we will assume that the scattering object is ergodic (e.g., Ref. [4]) and will replace averaging over time by averaging over a large statistically representative set of randomly created realizations of the multi-particle object. In other words, we will assume that $\langle\mathbf{S}(\mathbf{r}, t)\rangle=\operatorname{Re}[\langle\mathbf{S}(\mathbf{r})\rangle]=\overline{\mathbf{S}(\mathbf{r})}$ where the overbar denotes ensemble averaging and $\langle\ldots\rangle$ denotes averaging over a time interval much longer than the typical period of random fluctuations caused by chaotic movements of the particles.

For the sake of brevity, we will often refer to $\mathbf{E}(\mathbf{r})$ and $\mathbf{H}(\mathbf{r})$ as the electric and magnetic field, respectively, which is a common practice. It should be remembered, however, that these quantities represent only the time-independent (or slowly fluctuating) amplitudes of the actual timeharmonic physical fields.

\subsection{Target generation}

The targets used in the simulations consisted of a cylindrical volume of radius $R$ and axial length $L$. Within this volume are placed the origins of $N_{S}$ nonoverlapping spheres, each with identical radius $a$ and refractive index $\mathrm{m}$. The axis of the cylinder is taken to be the $z$ axis in a global coordinate system. The quantities $N_{S}$ and $L$ were varied in the simulations, whereas $R$ was set to a value sufficiently large, relative to both the sphere radius $a$ and $L$, so that the average Poynting vector, calculated in regions close to the target axis, was unaffected by the lateral boundary of the target. A maximum value of $L=10 a$ was used in the simulations, and for this length a target radius of $R=30 a$ to 40 a was sufficient to simulate plane-layer conditions.

For a set $R$ and $L$, the positions of the $N_{S}$ spheres in the target were generated via a Monte Carlo sampling procedure. Specifically, a trial sample of the cylindrical coordinates $\rho_{i}, \phi_{i}, z_{i}$ of sphere $i$ in the set is obtained according to

$\rho_{i}=R \mathcal{R}^{1 / 2}, \quad \phi_{i}=2 \pi \mathcal{R}, \quad z_{i}=z_{i-1}+\mathcal{R} \Delta z$, with

$\Delta z=\frac{4 a^{3}}{3 f R^{2}}$

in which $\mathcal{R}$ denotes a random number uniformly distributed between 0 and 1 (each occurrence of $\mathcal{R}$ is implied to be a new sample), and $f$ is the average volume fraction of the spheres. This procedure automatically constrains the volume fraction of the spheres in the target volume to be $f$. The trial obtained from Eq. (1) is now checked against the positions for sphere $i-1, i-2, \ldots$, and the trial is accepted if no overlap occurs between spheres and is rejected and resampled if overlap does occur. A starting value of $z_{1}=0$ is arbitrarily set, and the procedure is carried out to some value $N_{\text {samp }} \approx 1.5-2$ times $N_{S}$, where $N_{S}$ denotes the desired number of spheres in the target. The actual positions of the target spheres are then chosen as those of the topmost $N_{S}$ samples in the set; the rationale here is that any pair-correlation artifacts in sphere positions occurring at startup will be washed out in the topmost samples.

Fig. 1 shows a typical target configuration used in the simulations. The target has dimensions $R=40 a, L=10 a$, and contains $N_{S}=6000$ spheres; the corresponding volume fraction is $f=0.5$.

\subsection{Calculation procedure}

The Multiple Sphere $T$ Matrix (MSTM) code $[1,13]$ was used to calculate the electric $\mathbf{E}(\mathbf{r})$, magnetic $\mathbf{H}(\mathbf{r})$, and Poynting $\mathbf{S}(\mathbf{r})$ vector fields in and around a fixed multiparticle target for a sequence of sampled targets. Parameters held constant in the target sequence were the sphere size parameter and refractive index, the target radius $R$ and thickness $L$, and the number of spheres $N_{S}$; the latter constraint is equivalent to fixing the average volume fraction of the spheres in the target. The target was excited with a plane wave, propagating in the $x-z$ plane at an angle $\beta$ relative to the positive direction of the $z$ axis and linearly polarized either parallel or perpendicular to the $x-z$ plane.

The formulation employs a superposition representation of the electric field exterior to the spheres, that being

$\mathbf{E}_{e x t}(\mathbf{r})=\mathbf{E}_{i n c}(\mathbf{r})+\mathbf{E}_{s c a}(\mathbf{r})=\mathbf{E}_{i n c}(\mathbf{r})+\sum_{i=1}^{N_{s}} \mathbf{E}_{s c a, i}(\mathbf{r})$

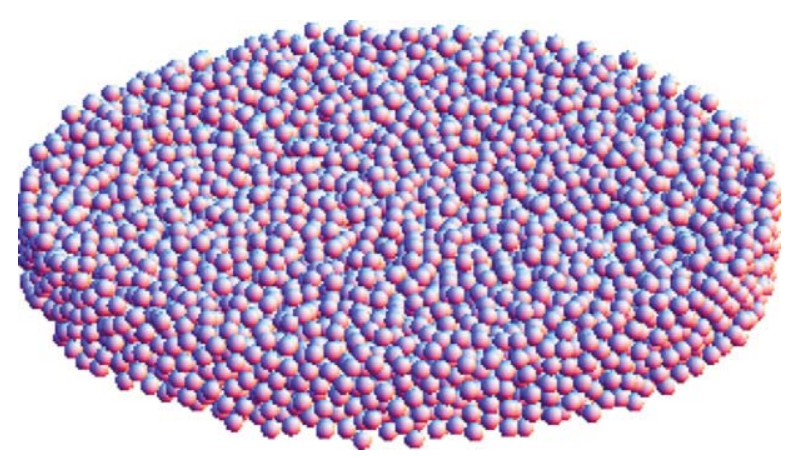

Fig. 1. Random sphere target. 
The scattered field, from a particular sphere, is described by outgoing vector spherical wave functions (VSWFs) centered about the origin of the sphere, and the incident field is described by a regular VSWF expansion centered about a common target origin:

$\mathbf{E}_{\text {inc }}(\mathbf{r})=\sum_{n=1}^{L_{T}} \sum_{m=-n}^{n} \sum_{p=1}^{2} f_{m n p} \mathbf{N}_{m n p}^{(1)}\left(\mathrm{k}_{0} \mathbf{r}\right)$

$\mathbf{E}_{s c a, i}(\mathbf{r})=\sum_{n=1}^{L_{S}} \sum_{m=-n}^{n} \sum_{p=1}^{2} a_{m n p}^{i} \mathbf{N}_{m n p}^{(3)}\left(\mathrm{k}_{0}\left(\mathbf{r}-\mathbf{r}_{i}\right)\right)$

In the above, $\mathrm{k}_{0}$ is the wavenumber in the external medium, $\mathbf{r}_{i}$ is the position vector of the center of the $i$ th sphere, and $\mathbf{N}_{m n p}$ denotes the VSWF of either type 1 (regular) or 3 (outgoing), of order $n$, degree $m$, and mode $p=1$ (TM) or 2 (TE). The truncation orders for the spheres, $L_{S}$, is set by inspection, i.e., by comparison of results computed with successively higher $L_{S}$. For the conditions examined here, $L_{S}$ was not dependent on the sphere concentration, and the required number of orders would depend solely on the size parameter and refractive index of the spheres. The truncation order for the incident field, $L_{T}$, will typically scale with the dimensionless distance $\mathrm{k}_{0}|\mathbf{r}|$ of the evaluation point from the target origin, and the incident field coefficients $f_{m n p}$ will depend on the direction and propagation state of the incident field.

The translation theorem for VSWFs, combined with the Mie relations for the sphere, results in a system of equations for the scattering coefficients $a_{m n p}^{i}$ :

$\frac{1}{\overline{a_{n p}}} a_{m n p}^{i}-\sum_{\substack{j=1 \\ j \neq i}}^{N_{S}} \sum_{l=1}^{L_{S}} \sum_{k=-l}^{l} \sum_{q=1}^{2} H_{m n p k l q}^{i-j} a_{k l q}^{j}=f_{m n p} e^{i k_{0}\left(x_{i} \sin \beta+z_{i} \cos \beta\right)}$

where $\bar{a}_{n p}$ are the Mie coefficients, which are functions of the sphere size parameter $\mathrm{k} a$ and refractive index $\mathrm{m}$, and $H^{i-j}$ is the outgoing translation matrix, which depends solely on the distance and direction of translation from origins $j$ to $i$.

Eq. (6) represents $2 N_{S} L_{S}\left(L_{S}+2\right)$ complex valued equations for the set of scattering coefficients. Iterative methods, combined with techniques to accelerate the translation operations, are employed in the MSTM code to generate a solution. All relevant microscopic and macroscopic electromagnetic properties of the target, i.e., external and internal field amplitudes, cross sections, scattering matrix elements, can be obtained analytically from the solution, and the predicted values are exact to the truncation error of the VSWF expansions. Of particular relevance here is the calculation of the complex electric and magnetic field vectors at arbitrary points. This is accomplished using the superposition of scattered and incident fields in Eqs. (3)-(5) for points exterior to the spheres, while at interior points, say within sphere $i$, the electric field is given by an expansion of regular VSWFs, evaluated in the sphere medium

$\mathbf{E}_{i n t, i}(\mathbf{r})=\sum_{n=1}^{L_{S}} \sum_{m=-n}^{n} \sum_{p=1}^{2} c_{m n p}^{i} \mathbf{N}_{m n p}^{(1)}\left(\mathrm{mk}_{0}\left(\mathbf{r}-\mathbf{r}_{i}\right)\right)$ in which the internal field coefficients $c_{m n p}^{i}$ are obtained from the scattering coefficients $a_{m n p}^{i}$ via the Mie relations.

\subsection{Configurational averaging}

The basic strategy of the calculations was to calculate and record electric and magnetic field amplitudes on a set of fixed sample points $\mathbf{r}_{p}, p=1,2, \ldots$, for a sequence of randomly generated sphere configurations, with each configuration corresponding to a set target geometry, sphere properties, and sphere volume fraction. The sample points are placed on a grid within a rectangular sample volume centered on the target axis. This sample volume, which is depicted in Fig. 2, has dimensions of $W \times W \times H$, where the lateral length $W$ is typically around $1 / 4$ to $1 / 2$ of the target radius $R$, and the axial height $H$ is set so the volume extends both above and below the top and bottom surfaces of the target.

The so-called coherent electric field, at a fixed sample point $\mathbf{r}_{p}$, is defined as the average of the electric field amplitude $\mathbf{E}(\mathbf{r})$ over a sufficiently long period of time. Assuming ergodicity, it can be obtained by averaging the calculated field amplitude values at the point over the sequence of random configurations, i.e.,

$$
\left\langle\mathbf{E}\left(\mathbf{r}_{p}\right) 》=\overline{\mathbf{E}\left(\mathbf{r}_{p}\right)}=\frac{1}{N_{c}} \sum_{k=1}^{N_{c}} \mathbf{E}\left(\mathbf{r}_{p}, c_{k}\right)\right.
$$

where $c_{k}$ denotes the parameters for configuration $k$ and $N_{c}$ is the number of sampled configurations. As explained in Ref. [14], the coherent electric field is not a real physical field since it is obtained by averaging the complex amplitude of the electric field rather than the electric field itself. The practical usefulness of this purely mathematical vector field and its magnetic analog will be clear from the following discussion.

Since the incident field propagates in the $x-z$ plane and, for a target of infinite lateral extent $(R \rightarrow \infty)$, the coherent field would be independent of $y$ - we would further refine the coherent field estimate by averaging over the sample points in the $y$ direction for a fixed $x_{p}, z_{p}$,

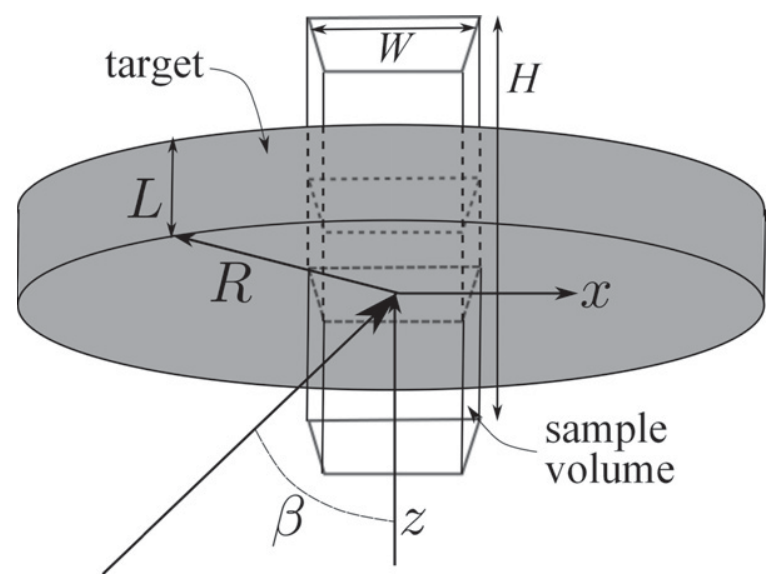

Fig. 2. Target and sampling configuration. 
via

$\overline{\mathbf{E}\left(x_{p}, z_{p}\right)}=\frac{1}{N_{p}} \sum_{y_{p}} \overline{\mathbf{E}\left(x_{p}, y_{p}, z_{p}\right)}$

where $N_{p}$ denotes the number of points in the $y$ direction. For the specific case of normal incidence $(\beta=0)$, the coherent field would depend solely on $z$, and averaging was performed over both the $x$ and $y$ directed sample points.

To avoid complicating the subsequent analysis, we will not distinguish between the normal and oblique incidence cases and their associated strategies for coherent field calculation: the coherent field at a point $\mathbf{r}$ will be denoted simply as $\overline{\mathbf{E}(\mathbf{r})}$, and it is implicitly understood that calculation of this quantity involves both configurational and spatial averaging. Given this, the electric field amplitude at a specific sample point $\mathbf{r}_{p}$, corresponding to a specific configuration $c$, can now be decomposed into the coherent and incoherent parts [5,6], via

$\mathbf{E}\left(\mathbf{r}_{p}, c\right)=\overline{\mathbf{E}\left(\mathbf{r}_{p}\right)}+\mathbf{E}^{\prime}\left(\mathbf{r}_{p}, c\right)$

where the ensemble average of $\mathbf{E}^{\prime}\left(\mathbf{r}_{p}, c\right)$ is zero. An analogous decomposition holds for the magnetic field at point $\mathbf{r}_{p}$ and configuration $c$.

The short-time average of the real-valued Poynting vector, at a sample point $\mathbf{r}_{p}$ and for a specific target configuration $c$, is given by

$\left\langle\mathbf{S}\left(\mathbf{r}_{p}, c, t\right)\right\rangle=\frac{1}{2} \operatorname{Re}\left[\mathbf{E}\left(\mathbf{r}_{p}, c\right) \times \mathbf{H}^{*}\left(\mathbf{r}_{p}, c\right)\right]$

The long-time average of this quantity at a point $r_{p}$ can be calculated per the strategy used to calculate the coherent field, i.e., assuming ergodicity and averaging over configurations and over the spatially independent direction in the sample volume. By the use of Eq. (10), the Poynting vector, averaged over a sufficiently long period of time, can be decomposed into a coherent part and a diffuse part $[5,6]$ :

$\overline{\left\langle\mathbf{S}\left(\mathbf{r}_{p}, c, t\right)\right\rangle}=\overline{\mathbf{S}\left(\mathbf{r}_{p}\right)}=\overline{\mathbf{S}_{c o h}\left(\mathbf{r}_{p}\right)}+\overline{\mathbf{S}_{d}\left(\mathbf{r}_{p}\right)}$

in which

$\overline{\mathbf{S}_{c o h}\left(\mathbf{r}_{p}\right)}=\frac{1}{2} \operatorname{Re}\left[\overline{\mathbf{E}\left(\mathbf{r}_{p}\right)} \times \overline{\mathbf{H}^{*}\left(\mathbf{r}_{p}\right)}\right]$
$\overline{\mathbf{S}_{d}\left(\mathbf{r}_{p}\right)}=\frac{1}{2} \operatorname{Re}\left[\overline{\mathbf{E}^{\prime}\left(\mathbf{r}_{p}, c\right) \times \mathbf{H}^{\prime *}\left(\mathbf{r}_{p}, c\right)}\right]$

The quantities that can be calculated directly, on the set of grid points $\mathbf{r}_{p}$, are the coherent fields $\overline{\mathbf{E}\left(\mathbf{r}_{p}\right)}$ and $\overline{\mathbf{H}\left(\mathbf{r}_{p}\right)}$, the average Poynting vector $\overline{\mathbf{S}\left(\mathbf{r}_{p}\right)}$, and the coherent flux $\overline{\mathbf{S}_{\text {coh }}\left(\mathbf{r}_{p}\right)}$. The scattering flux is obtained from Eq. (12).

\section{Results}

\subsection{General trends}

We begin by examining the general characteristics of the coherent field and the coherent and diffuse fluxes for targets exposed to oblique-incidence plane waves. The target, for this case, has a dimensionless radius $\mathrm{k}_{0} R=60$, dimensionless thickness $\mathrm{k}_{0} L=15$, and contains $N_{S}=2250$ spheres, each with size parameter $\mathrm{k}_{0} a=2$ and refractive index $\mathrm{m}=1.48$ (characteristic of quartz in the visible wavelengths); the average volume fraction for this case is $f=0.25$. Three orders were used to represent the scattered fields from the spheres, and the interaction equations in Eq. (6) contained 67,500 unknowns for each incident state and target configuration. The maximum order required to evaluate the incident field at points within the sample volume, via Eq. (4), was approximately $L_{T} \approx 60$. The MSTM code was run on the Auburn University College of Engineering compute cluster, using 64 processors, and complete solution for a set target configuration and incident angle (corresponding to two solutions to Eq. (6) for two mutually perpendicular incident polarizations) required around $15 \mathrm{~min}$. A total of 60 random configurations were used to compute the coherent fields and extinction and scattering fluxes.

Fig. 3 contrasts the structure of the local electric field amplitude for a particular configuration vs. that for the coherent field following configurational and spatial averaging. Both plots show levels of Re $\hat{\mathbf{y}} \cdot \mathbf{E}$ in the $x-z$ plane. The incident field has $\beta=30^{\circ}$, and is polarized perpendicular to the plane. Axes coordinates are $\mathrm{k}_{0} x$ and $\mathrm{k}_{0} z$, with the center of the target corresponding to the origin, and the target is contained within $\mathrm{k}_{0} z= \pm 7.5$. The coherent field on the right has been averaged over 60 configurations and over 21 planes in the $y$ direction evenly spaced between $\mathrm{k}_{0} y= \pm 20$.
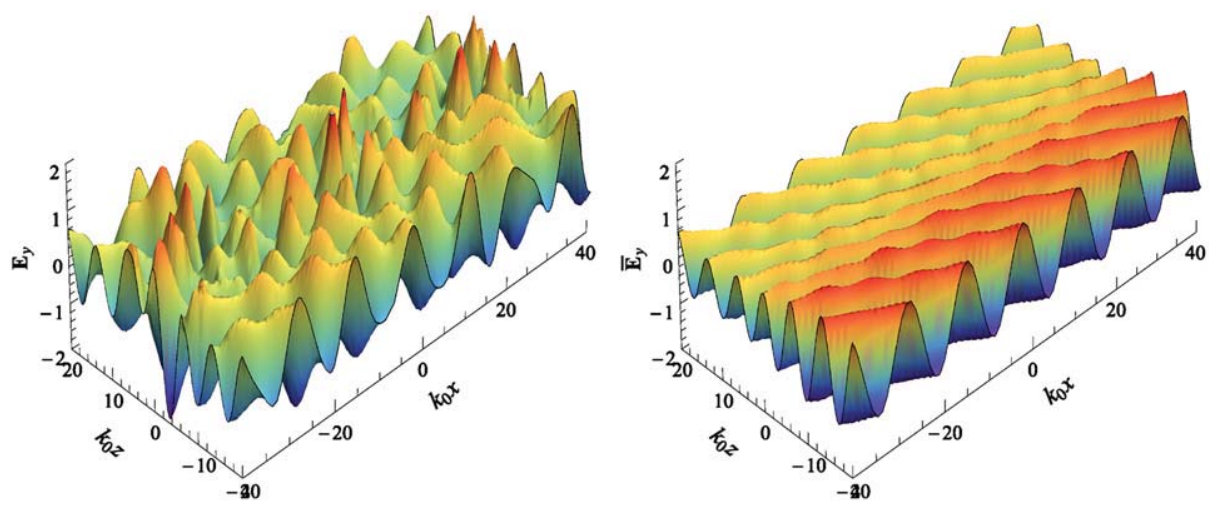

Fig. 3. Local value of $\operatorname{Re}(\hat{\mathbf{y}} \cdot \mathbf{E})$ (left), and coherent field $\operatorname{Re}(\hat{\mathbf{y}} \cdot \overline{\mathbf{E}})$. 

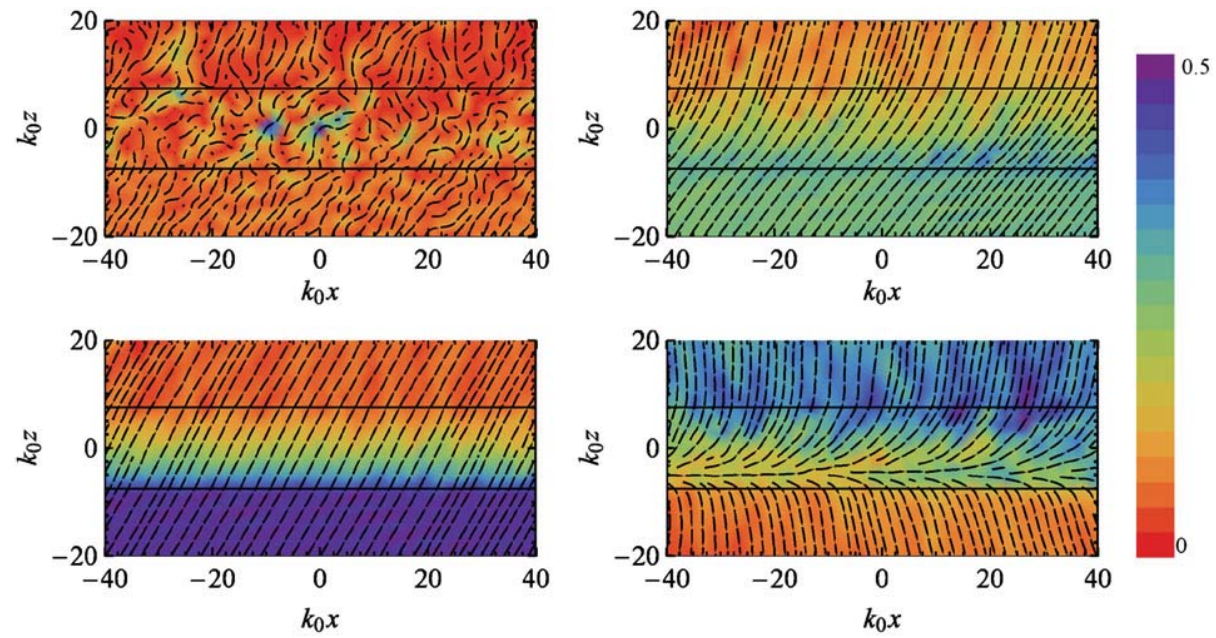

Fig. 4. Vector field of $\mathbf{S}$ in $x-z$ plane (top left), averaged (top right), and coherent (bottom left) and diffuse (bottom right) components of $\overline{\mathbf{S}}$. Shading represents magnitude of vector.

The coherent field in Fig. 3 contains characteristics associated with the reflection and transmission of a plane wave by a homogeneous layer. In particular, a refraction and retardation of the wave is evident as the field propagates through the target medium. This behavior is more apparent when the average Poynting vector is decomposed into coherent and diffuse parts. Shown in Fig. 4 are stream plots of the local Poynting vector in the $y=0, x-z$ plane for a fixed configuration, along with total average Poynting vector, and the coherent and diffuse contributions to the total average, for $\beta=30^{\circ}$ incidence and perpendicular polarization.

The two top plots in Fig. 4 display the directional flow of the total energy transfer in and about the target, with the left being a "snapshot" for a particular configuration, and the right showing the average flow pattern. The average flux entering the system is primarily in the direction of the incident beam, and it is steered toward the normal direction during the transport across the target. A simple radiative transport interpretation of this effect is that scattering by the particles results in an effective retardation of the energy flow, and the effect of this is to shift the overall direction of the flow toward the normal directions.

A more concrete interpretation, in our view, makes use of the principle of energy conservation. The spheres, for this specific example calculation, are nonabsorbing, and for such a situation $\overline{\mathbf{S}}$ must be divergence free at all points. Furthermore, for a target of infinite lateral extent, this condition would imply $\hat{\mathbf{z}} \cdot \overline{\mathbf{S}}=$ constant at all axial positions. Scattering by the spheres (and absorption, if present) act to extinguish the coherent field - as observed in Fig. 3 - and this results in a sink in the coherent flux per the definition in Eq. (13). Due to overall conservation of energy, this sink must reappear as a source in the diffuse flux. The two lower stream plots in Fig. 4 illustrate this interaction between coherent field destruction and diffuse flux (or, equivalently, incoherent field) formation. The coherent flux vector field behaves analogously to a plane wave interacting with a absorbing homogeneous layer, in which the effective absorption describes the destruction of the coherent field by scattering. Likewise, the diffuse flux is created within the target, and emerges from both the top and bottom surfaces.

\subsection{Effective medium model of coherent field}

We will now focus attention on the extinction of the coherent field within the medium, with the objective of identifying, via direct simulations coupled with modeling, the effective refractive index of the target. To simplify the presentation, we will restrict the examination to the normal incidence case $(\beta=0)$.

The effective medium theories of $[5,7]$ were based on a half-space model of the inhomogeneous medium - for which the regions $z<0$ and $z \geq 0$ consisted of free space and a random distribution of particles, respectively - that was excited by an $\hat{\mathbf{x}}$ polarized, unit amplitude plane wave propagating in the $+z$ direction. The coherent field, for this case, is a plane wave characterized by an effective propagation constant, i.e.,

$\overline{\mathbf{E}_{c o h}(z)}=\hat{\mathbf{x}} e^{i k_{e} z}$

The most straightforward estimation of the effective refractive index $\mathrm{m}_{e}=\mathrm{k}_{e} / \mathrm{k}_{0}$, from the simulation results, would therefore be obtained from

$\mathrm{m}_{e} \approx \frac{1}{\mathrm{ik}_{0} L} \ln \left(\frac{\hat{\mathbf{x}} \cdot \overline{\mathbf{E}_{c o h}(L)}}{\hat{\mathbf{x}} \cdot \overline{\mathbf{E}_{c o h}(0)}}\right)$

where $\overline{\mathbf{E}_{\text {coh }}(0)}$ and $\overline{\mathbf{E}_{c o h}(L)}$ are the calculated complex coherent field at the target boundaries.

The half space model of Eq. (15) neglects the existence of a downward-propagating component in the coherent field, which could arise from a reflection of the upwardpropagating component from the top boundary. To examine such effects, we performed a more detailed modeling of the coherent field based on a homogeneous layer (or film) of thickness $L_{e}$ and refractive index $\mathrm{m}_{e}$. The model 
formula for $\overline{\mathbf{E}_{\text {coh }}(z)}$ is given by

$\overline{\mathbf{E}_{c o h}(z)}=\hat{\mathbf{x}} \begin{cases}e^{i \mathrm{k}_{0} z}+r e^{-i \mathrm{k}_{0} z}, & z<0 \\ a e^{i \mathrm{k}_{e} z}+b e^{-i \mathrm{k}_{e} z}, & 0 \leq z<L_{e} \\ t e^{i \mathrm{k}_{0} z}, & z \geq L_{e}\end{cases}$

with

$r=\frac{1}{D}\left[\left(\mathrm{~m}_{e}^{2}-1\right)\left(1-e^{2 \mathrm{im}_{e} L_{e}}\right)\right]$

$a=-\frac{2\left(\mathrm{~m}_{e}+1\right)}{D}$

$b=-\frac{2\left(\mathrm{~m}_{e}-1\right) e^{2 \mathrm{im}_{e} L_{e}}}{D}$

$t=-\frac{4 \mathrm{~m}_{e} e^{i\left(\mathrm{~m}_{e}-1\right) L_{e}}}{D}$

and

$D=e^{2 \mathrm{im}_{e} L_{e}}\left(1-\mathrm{m}_{e}\right)^{2}-\left(1+\mathrm{m}_{e}\right)^{2}$

The coherent field model has three parameters, being the real and imaginary parts of $\mathrm{m}_{e}$ and the effective thickness $L_{e}$. We keep the thickness $L_{e}$ a parameter - as opposed to setting it equal to thickness $L$ used to generate the targets - because the target does not have sharp, distinct boundaries. That is, since all sphere origins must lie between $z=0$ and $L$, there will be a region, of thickness equal to the sphere radius, over which the medium transitions from inhomogeneous to homogeneous. Values of $\mathrm{m}_{e}$ and $L_{e}$, for a given set of coherent field simulation data, were obtained by a nonlinear least square error fit of Eq. (17) to the data. To perform this fit, the point $z=L_{e} / 2$ in Eq. (17) was made to coincide with the target center in the simulation data.

Simulations were performed using spheres with $\mathrm{k}_{0} a=2.645$ and $\mathrm{m}=1.194$, which correspond to the latex spheres-in-water experimental measurements conducted in [15] and examined theoretically in [16]. The target thickness and radius were $\mathrm{k}_{0} L=26.45$ and $\mathrm{k}_{0} R=79.35$ (i.e., 10 and 30 sphere radii), and volume fractions ranged from $f=0.01$ to 0.5 .

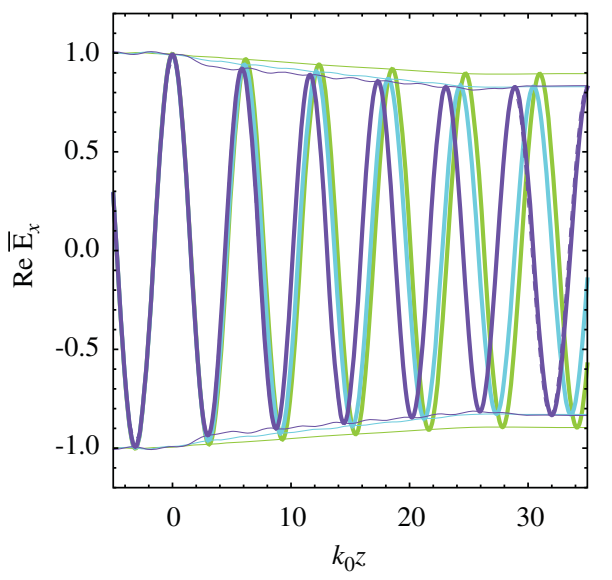

Results appear in Fig. 5, which show the coherent field phase and magnitude as a function of position, for four values of volume fraction $f$ ranging from 0.025 to 0.5 . The solid lines are the simulation results, and the dashed lines show the predictions of Eq. (17) using the fitted $\mathrm{m}_{e}$ and $\mathrm{k}_{0} L_{e}$ values for the data set. The simulation results and model predictions are presented so that $z=0$ represents the lower boundary of the slab, and the vertical colored lines, in the neighborhood of $\mathrm{k}_{0} z=25-30$, mark the fitted value of $\mathrm{k}_{0} L_{e}$ for the particular set. The phase results indicate the increasing retardation of the coherent wave as concentration increases; results for $f=0.025$ are not shown in this plot because they are negligibly different than those for $f=0.079$. The magnitude results, which are plotted on a log scale, show an exponential decrease in amplitude. Of particular interest are the oscillations in $|\overline{\mathbf{E}(z)}|$ seen for the relatively large $f$ values. This can be interpreted as an effect of interference between the downward and upwards propagating wave components that are present in a finite-thickness layer. The model results overestimate the amplitude of the downward component - likely because of the representation of the boundaries as sharp interfaces - yet the simulation results do reveal that a downward-propagating component is present in the coherent field.

The specific values of sphere size parameter and refractive index, used in the results of Fig. 5, were chosen to allow a comparison of the predicted attenuation coefficient $\alpha_{t}=2 \mathrm{k}_{0} \operatorname{Im}\left(\mathrm{m}_{e}\right)$ of the medium with that derived from effective medium theory, and measured by experiment, as described in [16]. A useful indicator of the packing density effects on attenuation is the ratio of the exact result to that predicted from radiative transfer theory, given by

$\gamma=\frac{\alpha_{t}}{3 f C_{e x t} /\left(4 \pi a^{3}\right)}=\frac{4 \operatorname{Im}\left(\mathrm{m}_{e}\right) \mathrm{k}_{0} a}{3 f Q_{e x t}}$

where $C_{\text {ext }}$ and $Q_{e x t}$ are the extinction cross section and extinction efficiency of the sphere. For sufficiently small $f$ this ratio should go to unity. Results are given in Fig. 6, which show attenuation ratios as a function of volume

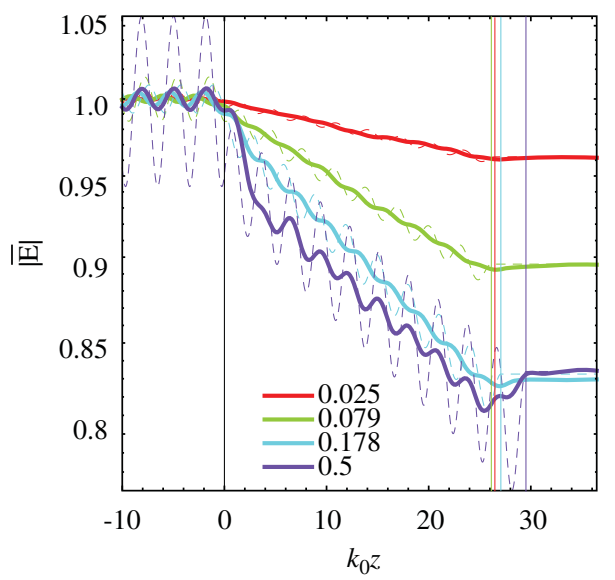

Fig. 5. Re $\hat{\mathbf{x}} \cdot \overline{\mathbf{E}_{\text {coh }}(z)}$ (left) and $\left|\overline{\mathbf{E}_{\text {coh }}(z)}\right|$ (right) vs. $\mathrm{k}_{0} z$, for $\mathrm{k}_{0} a=2.645$ and $\mathrm{m}=1.194$ and for different volume fractions. Solid line: simulations, dashed lines: model fit to Eq. (17). Colored vertical lines denote fit values of $\mathrm{k} L_{e}$. 


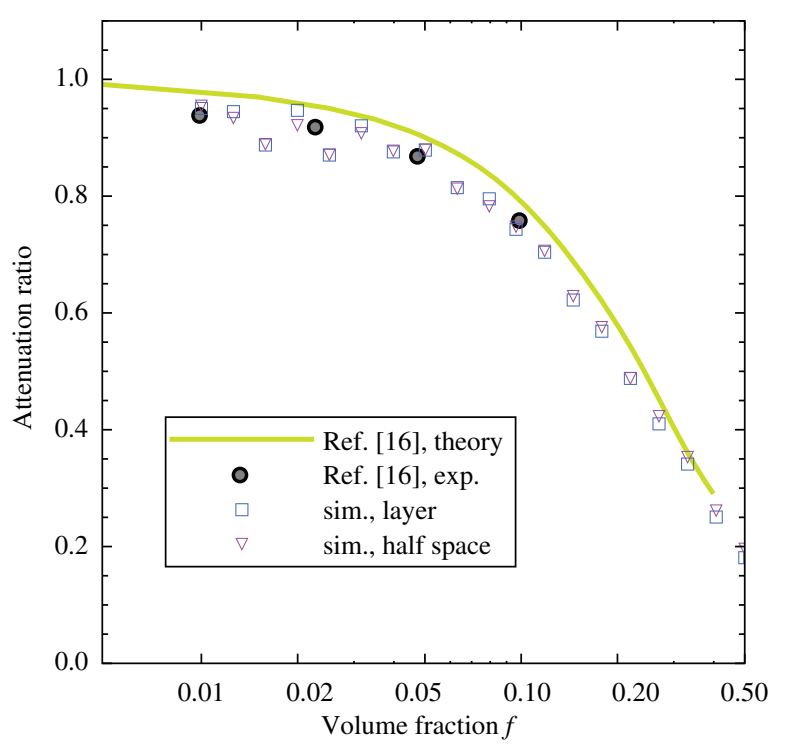

Fig. 6. Attenuation ratio $\gamma$ (Eq. (23)) vs. volume fraction $f$, for simulation results predicted by the layer model (Eq. (17)) and the half-space model (Eq. (16)), along with experimental and theoretical results of [16]. Sphere and target properties are the same as Fig. 4.

fraction $f$. Two sets of simulation results are presented, corresponding to $\alpha_{t}$ derived the simple plane wave model of Eq. (16) and the layer model of Eq. (17). Also appearing are the theoretical and experimental results reported in [16].

The results in Fig. 6 show that, for the specific conditions examined here, the attenuation coefficient can be accurately predicted by treating the target as a half space-in that the values of $\alpha_{t}$ derived from Eq. (16) are not significantly different than those obtained from a more comprehensive model. Furthermore, the simulation results are highly consistent with both effective medium theory and experiment, especially for $f$ greater than around 0.1. For smaller volume fractions the simulation results show a small degree of scatter around the asymptotic limit of unity. We suspect that this is an artifact of the averaging process, as a smaller number of spheres in the target - corresponding to smaller $f$ - leads to a relatively larger standard deviation in the configurationdependent properties of the target. Consequently, a number of configuration samples greater than the 100 used here for each point may be required to accurately resolve the coherent field properties, yet, at the same time, the simple, independent scattering model works quite well in this limit.

\subsection{The diffuse flux}

Per the discussion in Section 3.1, the divergence of the average Poynting vector $\overline{\mathbf{S}(\mathbf{r})}$ is zero for the nonabsorbing spheres examined here. The destruction of the coherent flux $\overline{\mathbf{S}_{\text {coh }}(\mathbf{r})}$ by "effective" absorption - as manifested in a nonzero imaginary part of the effective refractive index $\mathrm{m}_{e}$ - must be balanced by the creation of the diffusive flux $\overline{\mathbf{S}_{d}(\mathbf{r})}$.
Ishimaru et al. [17] applied the diffusion approximation to the radiative transport equation to model the diffuse intensity and flux in systems containing relatively large densities of scattering particles. It should be emphasized that the intensity - being, per the common radiative transport definition, a scalar quantity describing the flow of energy in a particular direction and contained within a differential solid angle - cannot be extracted from the simulations results [18]. That is, the only physically meaningful quantities obtained from the simulations are the electric and magnetic field amplitudes and the Poynting vector. In this sense, a comparison of a radiative transport equation solution for the diffuse intensity with a direct simulation, based on Maxwell's equations, is impossible. However, a radiative transport prediction of the diffuse flux, which is obtained from an integral of the diffuse intensity, can be compared with the simulation results.

To do this, we adapt the diffusion approximation developed in Ref. [17] to the conditions of the simulations. In particular, under normal incidence conditions, the diffuse flux will be parallel to the $z$ axis and will be a function solely of $z$. For the nonabsorbing conditions examined here (albedo of unity), the diffuse flux will be related to the average diffuse intensity $U(z)$ via,

$\overline{S_{d, z}(z)}=\frac{1}{1-g_{1}}\left(I_{0} g_{1} e^{-\alpha_{t} z}-\frac{4 \pi}{3 \alpha_{t}} \frac{d U(z)}{d z}\right)$

where $\overline{S_{d, z}(z)}=\hat{\mathbf{z}} \cdot \overline{\mathbf{S}_{d}(z)}, I_{0}$ is the intensity of the incident field ( $=1 / 2$ for the unit-amplitude incident electric field), and $g_{1}$ is the dimensionless scattering asymmetry parameter of the particles (i.e., a characteristic of the fraction of energy single-scattered by the particles into the forward directions). The average diffuse intensity, for nonabsorbing conditions, satisfies

$\frac{d^{2} U(z)}{d z^{2}}=-\frac{3 I_{0} \alpha_{t}^{2}}{4 \pi} e^{-\alpha_{t} z}$

with the boundary conditions,

$U(0)=-\frac{1}{2 \pi} \overline{S_{d, z}(0)}$

$U(L)=\frac{1}{2 \pi} \overline{S_{d, z}(L)}$

where the fluxes at the boundaries, $\overline{S_{d, z}(0)}$ and $\overline{S_{d, z}(L)}$, are related to $U$ via Eq. (24). The solution for the diffuse flux is obtained as

$\overline{S_{d, z}(z)}=I_{0}\left(\frac{5-e^{-\alpha_{t} L}}{4-3 \alpha_{t} L\left(1-g_{1}\right)}-e^{-\alpha_{t} z}\right)$

To compare the model with simulation results, we set $\alpha_{t}=2 \mathrm{k}_{0} \operatorname{Im}\left(\mathrm{m}_{e}\right)$ and $L=L_{e}$, where $\mathrm{m}_{e}$ and $L_{e}$ correspond to the values obtained, for a specific volume fraction $f$, by fitting the coherent field to the plane layer model. We then determined a value of the asymmetry parameter $g_{1}$, for each $f$, by minimizing the least square error between the simulation and diffusion model predictions of the diffuse flux.

Shown in Fig. 7 are the simulation and model predictions of the diffuse flux, as a function of dimensionless position $\mathrm{k}_{0} z$. For $f$ up to around 0.2 , the diffusion model 


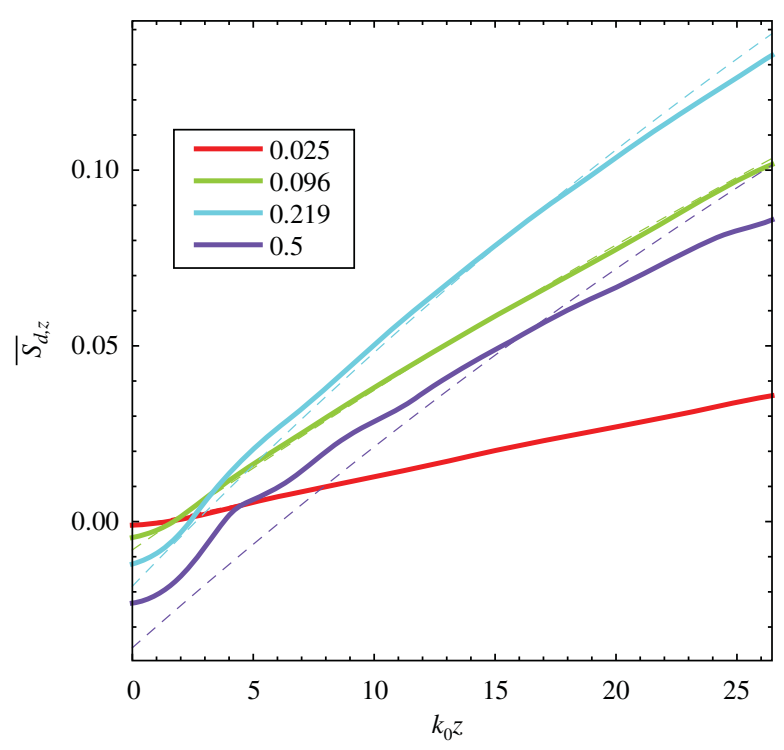

Fig. 7. Axial component of diffuse flux $\overline{S_{d z z}(z)}$ vs. dimensionless axial position $\mathrm{k}_{0} z$, with volume fraction $f$ as a parameter. Solid lines are simulation results, dotted are model predictions from Eq. (28).

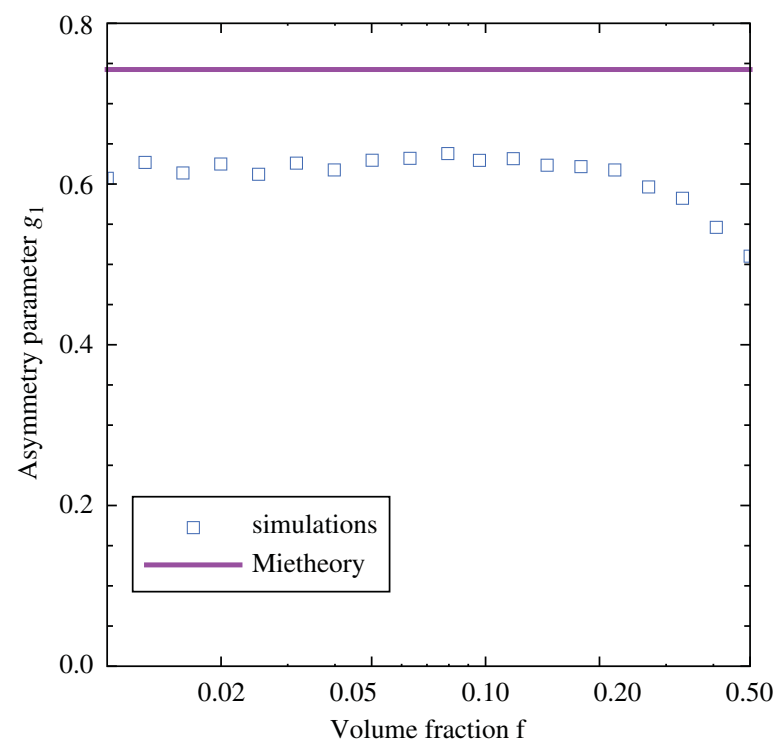

Fig. 8. Asymmetry parameter $g_{1}$, calculated from fitting of Eq. (28) to simulation results, vs. volume fraction $f$. The solid line is the single sphere Mie result.

does an excellent job of capturing the magnitude and distribution of the diffuse flux as calculated by the simulations. The simulation results for $f=0.5$, and to a lesser extent for $f=0.22$, show characteristics near $z=0$ that could not be represented by the exponential model of Eq. (28). It is interesting to note that the oscillations seen in the $f=0.5$ data have a wavelength roughly equal to twice the sphere size parameter, and this may point to an effect due to an emerging lattice structure among the spheres.
The fitted values of asymmetry parameter $g_{1}$ are shown in Fig. 8 as a function of $f$. The values of $g_{1}$ are fairly constant for $f$ up to around 0.2 , and are roughly 0.8 of the single-sphere Mie value. Undue emphasis should not be placed on the correspondence (or lack thereof) of the fitted $g_{1}$ results to the Mie value. The largest optical depth $\alpha_{t} L_{e}$ among the simulation results is 0.42 for $f=0.33$, and conditions for $f<0.1$ could be justifiably described as optically thin and would contradict the optically thick assumptions inherent in the diffusion approximation. What is more relevant, in regard to the results in Fig. 8, is the transition in $g_{1}$ from a constant, at low $f$, to a decreasing function of $f$. This behavior indicates an increasing effect, for large $f$, of pair-correlated positions among the particles, and is consistent with the results of Ref. [19] obtained using the structure-factor approach coupled with the Percus-Yevick approximation [20].

\section{Summary}

We have demonstrated that numerically exact solutions of Maxwell's equations in multiple sphere systems, combined with configurational and spatial averaging, can provide direct simulations of the coherent field and the coherent and diffuse energy fluxes that are entirely consistent with theoretical formulations of radiative transport in random, discretely inhomogeneous media. We should emphasize that the performed simulations are quite tractable on modern parallel computer platforms; the total computational time involved in this investigation was on the order of 10's of hours. Indeed, we will submit that direct simulations have a future role not as simply verifying radiative transfer theories, but rather as a practical means of calculating radiative transfer in high-concentration particle systems such as regoliths, pigment layers, deposits, etc.

\section{References}

[1] Mackowski DW, Mishchenko MI. A multiple sphere T-matrix Fortran code for use on parallel computer clusters. J Quant Spectrosc Radiat Transfer 2011;112:2182-92.

[2] Mishchenko MI, Liu L, Mackowski DW, Cairns B, Videen G. Multiple scattering by random particulate media: exact 3D results. Opt Exp 2007;15(6):

2822-36 URL 〈http://www.ncbi.nlm.nih.gov/pubmed/19550586〉.

[3] Mackowski DW, Mishchenko MI. Direct simulation of multiple scattering by discrete random media illuminated by Gaussian beams. Phys Rev A 2011;83(1). http://dx.doi.org/10.1103/PhysRevA. $83.013804013804-+$

[4] Mishchenko MI, Travis LD, Lacis AA. Multiple scattering of light by particles: radiative transfer and coherent backscattering. Cambridge, UK: Cambridge University Press; 2006.

[5] Ishimaru A. Theory and application of wave propagation and scattering in random media. IEEE Proc 1977;65:1030-61.

[6] Ishimaru A. Wave propagation and scattering in random media, vols. 1 and 2. Academic; 1978.

[7] Varadan VK, Bringi VN, Varadan VV. Coherent electromagnetic wave propagation through randomly distributed dielectric scatterers. Phys Rev D 1979;19:2480-9.

[8] Foldy LL. The multiple scattering of waves. i. General theory of isotropic scattering by randomly distributed scatterers. Phys Rev 1945;67(3-4):107-19. http://dx.doi.org/10.1103/PhysRev.67.107.

[9] Lax M. Multiple scattering of waves. ii. The effective field in dense systems. Phys Rev 1952;85(4):621-9. http://dx.doi.org/10. 1103/PhysRev.85.621. 
[10] Doicu A, Wriedt T, Eremin YA. Light scattering by systems of particles. Null-field method with discrete sources: theory and programs. Berlin: Springer; 2006.

[11] Tsang L, Mandt C, Ding KH. Monte Carlo simulation of the extinction rate of dense media with randomly distributed dielectric spheres based on solution to Maxwell's equations. Opt Lett 1992;17:314-6.

[12] Bringi VN, Varadan VV, Varadan VK. Coherent wave attenuation by a random distribution of particles. Radio Sci 1982;17:946-52.

[13] Multiple-sphere codes are available at <www.eng.auburn.edu/ users/dmckwski/scatcodes $>$.

[14] Mishchenko M, Tishkovets V, Travis L, Cairns B, Dlugach J, Liu L, et al. Electromagnetic scattering by a morphologically complex object: fundamental concepts and common misconceptions. J Quant Spectrosc Radiat Transfer 2011;112(4):671-92.
[15] Ishimaru A, Kuga Y. Attenuation constant of a coherent field in a dense distribution of particles. J Opt Soc Am A 1982;72(10):1317-20.

[16] Varadan VK, Bringi VN, Varadan VV, Ishimaru A. Multiple scattering theory for waves in discrete random media and comparison with experiment. Radio Sci 1983;18:321-7.

[17] Ishimaru A, Kuga Y, Cheung R, Shimizu K. Scattering and diffusion of a beam wave in randomly distributed scatterers. J Opt Soc Am 1983;73(2):131-6.

[18] Mishchenko M. Directional radiometry and radiative transfer: a new paradigm. J Quant Spectrosc Radiat Transfer 2011;112(13):2079-94.

[19] Mishchenko M. Asymmetry parameters of the phase function for densely packed scattering grains. J Quant Spectrosc Radiat Transfer 1994;52(1):95-110.

[20] Balescu R. Equilibrium and nonequilibrium statistical mechanics. NASA STI/Recon Technical Report A 76; 1975. p. 32809. 